\title{
Word Order and Inverse Voice in Isthmus Mixe
}

\author{
JULIA DIETERMAN
}

University of Texas at Arlington, SIL International

\section{Introduction}

Isthmus Mixe (IM) is an Mesoamerican language of the Mixe-Zoque family, spoken in southern Mexico in the state of Oaxaca. In the 1990 census there were reported to be 20,000 speakers (Grimes 1996:88). The major city is San Juan Guichicovi; the language is also known as Guichicovi Mixe.

IM is primarily an oral language, and largely undescribed, with no previous published studies of word order. The data analyzed in the present paper were collected mainly by tape recording native speakers during visits by the author to the area, beginning in 1994. Transcriptions were made by the author and edited with the assistance of native speakers. The author had previously worked on a closely related Mixe language for a number of years (see Van Haitsma and Van Haitsma 1976).

\section{Background}

Traditionally, linguists have accepted basic word order as a language universal, although some exceptions may exist. In this paper the term basic word order refers to the order of the primary arguments of the clause - Verb, Subject, and Object. Greenberg (1966:76) posits basic word order as a fundamental typological parameter and states that the majority of the world's languages have a dominant word order and variations on the dominant order. Steele (1978:595) comments that the variations are not necessarily "semantically identical. ... Variations on a basic word order commonly indicate some change in topic and/or focus."

However, Mithun (1987) questions whether all languages have a basic word order. She argues that for some languages the word order reflects pragmatic functions such as "newsworthiness", rather than syntactic ordering. In spite of this qualification, it is nonetheless desirable for linguists to attempt to discover a basic word order for a previously undescribed language. If a basic syntactic order can be established, then explanations for variations can be sought and general patterns discovered in relation to the basic order. 


\section{Julia Dieterman}

On the surface, the word order of IM appears to be quite variable, including SOV, SVO, VSO, OSVand OVS. An examination of what is said about the word orders of other Mixe-Zoque languages also shows considerable variability. Yasugi (1995:372-7) lists the basic word order of two Zoque languages as SVO and one as VOS. Four Mixe languages are listed: Sayula Popoluca as having free word order, Oluta Popoluca as SVO, and both Coatlán Mixe and Tlahuitoltepec Mixe as "VSO > SVO / SOV (a marked, non-basic order ...)"; it is unclear as to what is meant by the latter designation.

Campbell, Kaufman and Smith-Stark (1986:547-8) discuss the word order of Mixe languages in some detail and state that Tlahuitoltepec sentences are mainly VSO. ${ }^{1}$ Their relevant examples of Coatlán Mixe are SVO. IM is not included in these surveys since there are no previous published studies of IM grammar.

In this paper, I posit a basic word order of SOV and analyze IM as having an inverse voice category that is expressed by subject-object inversion, or OSV. I further claim that this inversion, taken together with discourse factors, explains the surface word order variation. Empirical evidence of the IM inverse voice will be adduced from morphological, syntactic, and pragmatic phenomena based on a quantitative analysis of five IM narrative texts.

The main corpus of data analyzed in this study consists of five folktaletype narratives. ${ }^{2}$ The corpus contains a total of 521 clauses, of which 216 are transitive, 241 are intransitive, 36 are verbless, and 28 imperative. Of the 216 transitive clauses, 150 are third person (3P) Subject and 3P Object (3P-3P), an important subcategory to be discussed below. Direct quotations are included in the study corpus, unless otherwise indicated. There is no difference between narrative clauses and direct quotation clauses for the phenomena in the topics under study. Some aspects of the analysis require using direct quotations for first person (1P) and second person (2P) arguments (Section 2.2.1).

\section{Grammatical voice}

\subsection{Definition of Inverse Voice}

Based on classical languages such as Sanskrit, Greek, and Latin, the grammatical categories of voice, typically based on inflections of the verb, have traditionally been labeled as active, middle, and passive. These were considered to be universal categories (Fox and Hopper 1994:ix). More recently, the category of inverse has been recognized as a distinction of voice in some languages with nominativeaccusative case systems. In distinguishing voice types, Givón (1994:8) identifies the active-direct voice as the unmarked standard in which the Agent is more topical than the Patient. The reverse is true of inverse voice, i.e. the Patient is more

\footnotetext{
${ }^{1}$ Campbell, Kaufman and Smith-Stark (1986:547) state that the SOV word order found in Tlahuitoltepec Mixe and Coatlán Mixe is a "marked, non-basic order when the particle $t \dot{t}$ 'already' (perfective) is present." In Isthmus Mixe there are numerous examples of SOV without the particle $t \dot{t}$ 'already', as well as examples in which it occurs.

${ }^{2}$ The five narratives are named Armadillo (A), Cat and Bird (C\&B), Country Boy (CB), Orphan (O), Poor Man (PM).
} 
Word Order and Inverse Voice in Isthmus Mixe

topical than the Agent. In neither voice is the less topical entity suppressed or demoted. In passive voice, in contrast, the Patient is much more topical than the Agent, which is either suppressed or demoted. And in the antipassive voice, found in some ergative languages, the Agent is much more topical than the Patient.

Although inverse voice is not grammatically encoded in English, it can be represented by fronting the Patient (sometimes called Y-movement or L-dislocation) to show topicality. The following examples illustrate the distinctions between active, passive and inverse voice in English.

(1) Active voice: Peter invited Alice to the party.

(2) Passive voice: Alice was invited to the party (by Peter).

(3) Patient fronting (inverse): (As for) Alice, Peter invited her to the party.

The normal expectation is for the action to proceed from the Agent, Peter, to the Patient, Alice, as in example (1); this is the direct-active voice. In the passive voice the Agent is demoted or suppressed along with the notion of a flow of action. If one desires only to demote rather than suppress the Agent in the passive, the by plus Agent may be used as shown in parentheses in example (2). In example (3), which represents inverse voice, the Patient, Alice, is marked as more topical by fronting yet Peter remains the Agent of the clause.

Passive voice does not occur in the present corpus of IM texts, and antipassive is not found since IM is a Nominative-Accusative language. The focus of this study, therefore, is the contrast between IM direct-transitive clauses and inversetransitive clauses.

\subsection{Pragmatic and Semantic Inverse Clauses}

Givón (1994) identifies two types of inverse clauses: pragmatic and semantic. Semantic inversion is related to agency hierarchy. Pragmatic inversion is related to the topicality or thematic importance of the Subject and Object. IM has both pragmatic and semantic inverse-transitive (INV-TR) clauses.

\subsubsection{Semantic inversion}

As described by Givón (1994), obligatory semantic inversion is associated with agency hierarchy or saliency, e.g. $1 \mathrm{P}>2 \mathrm{P}>3 \mathrm{P}$. The direct-transitive (DR-TR) clause is used when the Subject outranks the Object in the hierarchy, and the INV-TR clause is used when the Object outranks the Subject. When semantic inversion is present in a language, the speaker or writer is not given a choice regarding which forms to use; rather, the ranking hierarchy governs their use.

In IM, the agency hierarchy ranking of the participants is: $1 \mathrm{P}$ is greater than $2 \mathrm{P}$, and both of these are greater than $3 \mathrm{P}$. Semantic INV-TR clauses are distinguished morphologically from DR-TR clauses by a different set of person-agreement prefixes on the verb. The $1 \mathrm{P}$ and $2 \mathrm{P}$ INV-TR prefix is $f^{j}$-and the DR-TR 


\section{Julia Dieterman}

prefixes are $n$ - ' $1 \mathrm{P}^{\prime}$ and $m$ - ' $2 \mathrm{P}$ '. The semantic INV-TR clause in example (4) shows the Object 1P/2P prefix $\mathcal{S}^{j}$ - on the verb. The 1P suffix $-\overline{t s}^{j}$ on the negative morpheme $k a^{P} a$ co-references the agreement marker on the verb.

(4) Semantic inverse-transitive clause

$k a^{2} a-t^{j} \quad s^{j}-t_{s} u^{2} u t_{s}{ }^{j}$

NEG-1P 1P/2P_INV-eat meat-CL ${ }^{3}$

'Don't eat me.' (C\&B 20)

In contrast, the DR-TR clause, example (5), utilizes the Subject 1P prefix $n$ on the verb. The context indicates that the Object of the Verb is the addressee.

(5) Direct-transitive clause

$$
\begin{aligned}
& n-\overline{\mathbb{E}} u^{2} \overline{\boldsymbol{E}}-a{ }^{\prime} m-b^{j} \\
& \text { 1P-eat_meat-FUT-CL now } \\
& \text { 'I'm going to eat [you] now.' (C\&B 15) }
\end{aligned}
$$

The contexts of both examples indicate that the Subject in (4) and the Object in (5) is 2P. A NP or pronoun expression of either Subject or Object is not required when the referents are already identifiable from the context.

Since only one argument can be marked on the verb, whenever there is either a 1P Subject or a 1P Object, then $1 \mathrm{P}$ is marked on the verb. Likewise, 2P Subject or $2 \mathrm{P}$ Object ranks higher than 3P. When the higher ranking person is the Subject, the DR-TR clause is required. When the higher ranking person is the Object, the INV-TR clause is required. Semantic inversion is thus an obligatory grammatical feature of the language.

\subsubsection{Pragmatic inversion}

Pragmatic inversion differs from semantic inversion in several ways. According to Givón (1994), pragmatic inversion may be employed when both Subject and Object are 3P. The decisive factor in the choice of either the direct voice or the inverse voice is the relative topicality of Subject and Object. Topicality is defined by Givon as thematic importance in the discourse. Thus the DR-TR clause is used when the Subject is more topical than the Object, and the INV-TR clause is used when the Object is more topical than the Subject. When pragmatic inversion is utilized, the speaker or writer chooses to present the Object as more topical based on discourse considerations.

According to Payne (1994:316) "a more topical participant is more agentive, more animate, more individuated, more important either locally or globally, and/or more predictable in the sense that it may have already been referred to

\footnotetext{
${ }^{3}$ Abbreviations used in examples include the following: BEN benefactive, CL clause marker, INV inverse voice marker, FUT future tense marker, NEG negative.
} 
within the discourse context at hand." Examples (6) and (7) illustrate some of these indicators of topicality in IM.

Example (6) is a pragmatic INV-TR clause. The Subject is $P u \rho^{j} p$ 'alligator' and the Object is ha kra' 'the guy'. In addition to Subject-Object inversion, the clause is marked morphologically by an inverse suffix $-\dot{i}$ on the verb.(6) Pragmatic inverse-transitive clause

$$
\begin{aligned}
& \text { manit ha kra' Pus }{ }^{j} p \quad{ }^{j}-m a^{h} t \bar{s}-\dot{t} \\
& \text { then the guy alligator 3P-grab-INV } \\
& \text { 'Then [an] alligator grabbed the guy.' (O 11) }
\end{aligned}
$$

That the Object is more topical than the Subject in (6) is supported a priori by the fact that the Object is human and the Subject is an animal. Moreover, the Object has been previously introduced in the discourse as an orphan boy by means of a major-character type of introduction. The alligator is not introduced at all; it just happens to be in the water where the guy is bathing. Finally, the Object, the guy, is more important globally, because the story continues about him after the alligator dies. Therefore, since both Subject and Object are $3 \mathrm{P}$ and the Object is more topical, the INV-TR clause type is used.

In contrast to example (6), the Subject in the DR-TR clause in example (7) is more topical than the Object. The word order is SOV and it lacks the inverse marker suffix $-i$ on the verb.

Direct-transitive clause

$$
\begin{aligned}
& \mathrm{S} \quad \mathrm{V} \\
& \text { manit ha ts ji:d ha mu:j o-math }{ }^{h} \bar{s}^{j} \\
& \text { then the cat the bird 3P-grab-CL } \\
& \text { '... then the cat grabbed the bird.' (C\&B 13) }
\end{aligned}
$$

The Subject, ha ts ${ }^{j} i d$ 'the cat', in this instance is more topical than the Object, ha mu'j 'the bird'. The cat is more agentive in this sentence, and it is predictable from real-world knowledge that a cat will catch a bird. In addition, it is important to the events that follow that the cat does catch the bird. Therefore, the clause is direct with the action flowing from the Subject to the Object.

In pragmatic inversion the speaker or writer shows which participant is more topical in a particular situation by the use of DR-TR or INV-TR clauses. In another event in this narrative in which the bird outwits the cat, the bird is shown to be more topical than the cat.

In summary, there is pragmatic, morphological, and syntactic evidence for pragmatic inversion in IM. The pragmatic INV-TR clause is used when the Object is higher in topicality than the Subject. It utilizes the suffix $-i$ on the verb which indicates inverse voice, and the NP Object precedes the NP Subject, resulting in OSV order. In contrast, the DR-TR clause is used when the Subject is higher in 
Julia Dieterman

topicality than the Object. It lacks inverse voice morphology on the verb and the word order is SOV.

\section{Frequency of Explicit Subjects and Objects}

Further evidence of the Object's higher topicality in INV-TR clauses can be found in the quantitative distributions of clause types. In IM, the explicit NP expression of Subjects and Objects may be omitted when the discourse context is adequate to identify the participants. The majority of transitive clauses do not use NPs to designate both Subject and Object. Because the Object is higher in topicality in INV-TR clauses, the Subject is the argument most often omitted.

Table 1 shows the explicit arguments of each category found in all the $3 \mathrm{P}-3 \mathrm{P}$ transitive clauses in the narrative corpus. The display is restricted to $3 \mathrm{P}$ in order to show the contrast between the pragmatic inverse voice and the direct voice. The percentage of each word order is given, with the number of tokens below the percentage in parentheses.

Table 1: Word order variants of inverse-transitive (INV-TR) and direct-transitive (DR-TR) clauses in Isthmus Mixe

\begin{tabular}{|l|c|c|c|c|c|c|c|c|c|}
\hline & OV & VO & OSV & OVS & SV & SOV & SVO & VSO & V \\
\hline INV-TR & $\mathbf{6 4 \%}$ & $3 \%$ & $3 \%$ & $3 \%$ & $\mathbf{5 \%}$ & $\mathbf{X}$ & X & X & $23 \%$ \\
\hline (tokens) & $\mathbf{( 2 5 )}$ & $(1)$ & $(1)$ & $(1)$ & $\mathbf{( 2 )}$ & & & & $(9)$ \\
\hline \hline DR-TR & $\mathbf{1 8 \%}$ & $4 \%$ & $\mathrm{X}$ & $\mathrm{X}$ & $\mathbf{2 6 \%}$ & $\mathbf{2 8 \%}$ & $7 \%$ & $1 \%$ & $16 \%$ \\
\hline (tokens) & $\mathbf{( 2 0 )}$ & $(4)$ & & & $\mathbf{( 2 9 )}$ & $\mathbf{( 3 1 )}$ & $(8)$ & $(1)$ & $(18)$ \\
\hline
\end{tabular}

Three columns in Table 1 are in bold type. These are the most significant comparisons between INV-TR and DR-TR clauses related to the presence of explicit NP Subjects and Objects. In the first column, OV word order occurs in $64 \%$ of the INV-TR clauses but in only $18 \%$ of the DR-TR clauses. In contrast, SV occurs in only $5 \%$ of the INV-TR clauses but in $26 \%$ of the DR-TR clauses. Finally, SOV is the favored word order in DR-TR clauses at $28 \%$. These findings provide additional evidence that the Object is more topical in INV-TR clauses and the Subject is more topical in DR-TR clauses in IM.

\section{Referential Distance and Topic Persistence of Subjects and Objects}

Givón (1983) introduced a method of measuring topic continuity in discourse as an indirect means of determining the topicality of grammatical devices. In this analysis of the IM data, Givón's method was employed to measure the topicality of Subjects and Objects, using the measurements of Referential Distance (RD) and Topic Persistence (TP). 
$\mathrm{RD}$ is a look-back measurement of the distance between a particular referent or topic and its most recent previous mention in the discourse. RD is also referred to as anaphoric accessibility or the cognitive accessibility of the referent in relationship to prior mention in the text (Givón 1994:9). Following Givón's method, numerical values were assigned and the averages were obtained. The lower the $\mathrm{RD}$ average, the more continuity or topicality is indicated.

TP is a look-ahead measurement of the persistence of a particular referent in the subsequent discourse. TP is also described as cataphoric persistence or how long the referent remains in the following text. The higher the TP average, the greater is the continuity or topicality. This is in contrast to the RD values, in which lower averages indicate greater continuity. ${ }^{4}$

Table 2: Average Referential Distance (RD) and Topic Persistence (TP) values in Isthmus Mixe transitive clauses

\begin{tabular}{|l|c|c|c|c|}
\hline Clause Type & RD Subject & RD Object & TP Subject & TP Object \\
\hline DR-TR 3-3 & 1.9 & 4.6 & 1.9 & 1.5 \\
\hline INV-TR 3-3 & 1.9 & 3.8 & 1.5 & 3.4 \\
\hline
\end{tabular}

Table 2 shows the averages of RD and TP values in the IM 3P-3P transitive clauses. The most significant contrasts are shown by the average RD value of the DR-TR Subject as 1.9, and the RD value of the Object as 4.6, a difference of 2.7 value points. This is as expected, with the lower RD value showing the higher topicality of the Subject in the DR-TR clause.

In the TP measurement, the value for the INV-TR Subject is 1.5 and for the Object it is 3.4, a difference of 1.9 value points. According to the definition of TP, higher value indicates higher topicality. Therefore, the Object is higher in topicality than the Subject in INV-TR clauses. As it turns out, the exact same ratio is seen in the contrast between the TP DR-TR Object and the TP INV-TR Object; therefore the INV-TR Object is higher in topicality than the DR-TR Object.

Other values have smaller differences, which are, however, in the predicted direction. The average RD value of the INV-TR Object is 0.8 points lower than the RD value of the DR-TR Object, which indicates higher topicality of the INVTR Object in comparison to the DR-TR Object. In the TP values, the DR-TR Subject is 0.4 points higher than the Object, with the TP higher value indicating higher topicality. The one comparison which does not follow the predicted direction is the RD value of the Subject in INV-TR clauses as 1.9 and the Object as 3.8. The difference of 1.8 value points indicates a higher topicality for the Subject. Apart from this one instance, the results indicate the higher topicality of INV-TR Object and the higher topicality of the DR-TR Subject.

${ }^{4}$ See Givón 1983 for further description of the method for calculating RD and TP. 


\section{Julia Dieterman}

Although the primary purpose of this analysis is to determine the topicality of the Object in relationship to INV-TR clauses, another interesting result is evident. The averages of the RD and TP Subject values are similar for DR-TR clauses and INV-TR clauses. This result shows that the Subjects of INV-TR clauses are not demoted, as they would be in passive clauses, and is a further indicator that inverse voice is the correct interpretation of the data.

\section{Basic Word Order}

\subsection{Word order in DR-TR clauses}

Having shown that IM has an inverse voice construction that affects word order, we are now able to resolve the question of the basic word order in IM. Over onefourth of the 3P-3P transitive clauses in the corpus examined in this study are inverse voice; these clauses must be separated out from the direct-voice clauses to establish the language's basic word order. The word orders of DR-TR clauses in Table 1 show that IM is clearly an SOV language on grounds of frequency of occurrence. SOV is statistically the most frequent word order, occurring in $28 \%$ of the DR-TR 3P-3P clauses. Compatible with the SOV order are the one-argument clauses of SV (26\%) and OV (18\%). These three, SOV, SV, and OV, total $72 \%$ of the DR-TR clauses. The Verb alone is also consistent with SOV inasmuch as it does not contradict it.

Variations from the basic word order allow one or both of the NP arguments to follow the verb. These amount to $12 \%$ of the total of the DR-TR 3P-3P clauses, and reflect discourse considerations such as emphasis on the Verb when in sentence initial position, first mention or introduction of a postposed argument, or indicating something unusual happening to the postposed argument.

\subsection{Word Order in Main-line Clauses}

In analyzing basic word order, one parameter not often discussed is that of discourse type. Longacre (1995:333-5) claims that main-line clauses of narrative discourse are the most appropriate to use when establishing the basic word order of a language. Main-line (also called event-line) clauses are those which advance the chronology of the narrative (in contrast to background or off-line clauses). Givón (1994:155) states that "A prototypical transitive event involves an agent with full control, an affected patient, and an active verb form." The main-line clauses in the IM narrative corpus conform to Givón's description of prototypical transitive events more closely than the clauses of the 3P-3P clause corpus taken as a whole. Thus the parameters are more limited for main-line clauses than those used in this study thus far.

Of 79 main-line transitive clauses, $75 \%$ (59 tokens) are DR-TR and 25\% (20 tokens) are INV-TR, a ratio comparable with the distribution of the two clause types in the corpus as a whole. As shown in Table 3, in the DR-TR 3P-3P mainline clauses, the percentages of SOV and SV clauses (bold type) are even higher than in the entire DR-TR 3P-3P corpus. There are also fewer marked clauses of 
Word Order and Inverse Voice in Isthmus Mixe

SVO and VO. Thus the main-line clauses provide even stronger support for the claim that the basic word order of IM narrative texts is SOV.

Table 3: Comparison of word order in main-line direct-transitive (DR-TR) clauses with all DR-TR 3P-3P clauses in Isthmus Mixe narrative texts

\begin{tabular}{|c|c|c|c|c|c|c|c|}
\hline \multirow{3}{*}{$\begin{array}{c}\text { Main-line } \\
\text { DR-TR }\end{array}$} & SOV & SV & $\mathrm{OV}$ & $\mathrm{V}$ & SVO & $\mathrm{VO}$ & VSO \\
\hline & $32 \%$ & $34 \%$ & $14 \%$ & $14 \%$ & $5 \%$ & $2 \%$ & 0 \\
\hline & (19) & (20) & (8) & (8) & (3) & (1) & 0 \\
\hline \multirow{2}{*}{$\begin{array}{c}\text { All 3P-3P } \\
\text { DR-TR }\end{array}$} & $28 \%$ & $26 \%$ & $18 \%$ & $16 \%$ & $7 \%$ & $4 \%$ & $1 \%$ \\
\hline & (31) & (29) & (20) & (18) & (8) & (4) & (1) \\
\hline
\end{tabular}

\section{Translated materials}

Of the clauses analyzed in this study, $28 \%$ contain two explicit NP arguments. Considerably more clauses with two explicit NP arguments occur in complex sentences in translated materials. These translations often contain concepts that are uncommon or completely unknown in Mixe society. Since it cannot be assumed that the reader or listener will understand the general context, both DR-TR and INV-TR clauses tend to contain more explicit arguments than in the data used in this study. A pragmatic INV-TR clause from a narrative text translated from the New Testament is shown in example (8). Both Subject and Object arguments are explicit for the sake of clarity.

(8) Pragmatic inverse-transitive clause

$$
\begin{aligned}
& \text { O S V } \\
& \text { maniti Elisabet ha Dios-Pespiritu }{ }^{j}-n i-m e^{h} \overline{t s-i} \\
& \text { then Elizabeth the God-spirit 3P-BEN-arrive-INV } \\
& \text { 'Then Elizabeth was filled by the Holy Spirit.' (Luke 1:41b). }
\end{aligned}
$$

This sentence is considered well formed by native IM speakers. As discussed in Section 2.2.2, Subject-Object inversion results in OSV word order. Note that the usual English translation of the sentence is passive. The explictness of the translation lends additional evidence to support the claim of basic SOV word order and the inverse voice OSV word order in IM.

\section{Conclusion}

In this study, I have argued that IM is basically an SOV language with an inverse voice construction which produces the INV-TR clause variation of OSV. The shift of the Object to initial position reflects the greater topicality of the Object in INV-TR clauses, contrasting with DR-TR clauses in which the Subject is more topical. 


\section{Julia Dieterman}

Results of two quantitative analyses of the corpus of narrative data revealed the higher topicality of the Object in INV-TR clauses. In the first analysis, the Object was explicit in most INV-TR clauses, while the Subject was usually implicit. The ellipsis of the Subject implies higher topicality of the Object. In the second analysis, the Referential Distance and the Topic Persistence values for Subjects and Objects in INV-TR clauses showed higher topicality for Objects compared to the values for DR-TR clauses.

Since $26 \%$ of the $3 \mathrm{P}-3 \mathrm{P}$ transitive clauses in the corpus examined in this study are inverse voice, these clauses must be separated out from the direct voice clauses to establish the basic word order of the language. When this is done, SOV emerges clearly as the dominant word order in IM. By distinguishing carefully between grammatical voice categories, and recognizing variations based on discourse factors, it is possible to identify a basic SOV word order on the basis of frequency of occurrence for IM narrative texts.

Previous surveys and studies of Mixe languages have not revealed a language of the SOV word order type. Indeed, Campbell, Kaufman and Smith-Stark (1986:547-8) claim that there are no SOV languages in the Mesoamerican area. Similarly, Yasugi (1995:158) states that "Mesoamerica completely lacks SOV languages at present." However, neither Campbell et al. nor Yasugi had data on IM for their surveys. Yasugi (1995:158) notes, however, that "Mixe-Zoquean is a good candidate for SOV." The present study shows that Yasugi's intuition was correct. Once the inverse-direct voice distinction is recognized, and discourse considerations are taken into account, IM shows clear evidence of a basic SOV word order.

To my knowledge, an inverse voice category has not yet been posited for any other Mixe language. It is possible that closer inspection of such languages might likewise reveal voice and discourse distinctions that would help clarify their basic word orders.

\section{References}

Campbell, Lyle, Terrence Kaufman and Thomas C. Smith-Stark. 1986. MesoAmerica as a linguistic area. Language 62.3: 530-70.

El Nuevo Testamento en Mixe de Guichicovi. 1988. Mexico, D.F.: Liga del Sembrador, A.C.

Dieterman, Julia. 1998. Participant reference in Isthmus Mixe narrative discourse. Journal of Translation and Textlinguistics 10: 47-79.

Fox, Barbara and Hopper, Paul J. (eds.). 1994. Voice: Form and function. Amsterdam: Benjamins.

Givón, Talmy (ed.). 1983. Topic continuity in discourse: A quantitative crosslanguage study. Amsterdam: Benjamins. 
Givón, Talmy. 1994. The pragmatics of de-transitive voice: Functional and typological aspects of inversion. In Talmy Givón (ed.). 1994. Voice and inversion, 3-44. Amsterdam: Benjamins.

Greenberg, Joseph H. 1966. Some universals of grammar with particular reference to the order of meaningful elements. In Joseph H. Greenberg (ed.). Universals of Language, 2nd edition, 73-113. Cambridge, MA: MIT Press.

Grimes, Barbara F (ed.). 1996. Ethnologue: Languages of the world, 13th edition. Dallas: SIL.

Longacre, Robert E. 1995. Left shifts in strongly VSO languages. In Pamela Downing and Michael Noonan (eds.). Word order in discourse, 331-354. Amsterdam: Benjamins.

Mithun, Marianne. 1987. Is basic word order universal? In Russel S. Tomlin (ed.). Coherence and Grounding in Discourse, 281-328. Amsterdam: Benjamins.

Payne, Doris. 1994. The Tupí-Guaraní inverse. In Barbara Fox and Paul J. Hopper, (eds.). Voice: Form and function, 313-40. Amsterdam: Benjamins.

Steele, Susan. 1978. Word order variation: A typological study. In Joseph H. Greenberg (ed.). Universals of human language, Vol 4, Syntax, 585-624. Stanford: Stanford University Press.

Van Haitsma, Julia Dieterman and Willard Van Haitsma. 1976. A hierarchical sketch of Mixe as spoken in San José El Paraiso. Norman, OK: Summer Institute of Linguistics [Summer Institute of Linguistics Publications in Linguistics and Related Fields 44].

Yasugi, Yoshiho. 1995. Native middle American languages: An areal-typological perspective. Osaka, Japan: National Museum of Ethnology.

\author{
Julia Dieterman \\ P.O. Box 381153 \\ Duncanville TX 75138 \\ julia_dieterman@sil.org
}

